\title{
Oxidation softens mantle rocks
}

Seismic waves that propagate through a layer of Earth's upper mantle are highly attenuated. Contrary to general thinking, this attenuation seems to be strongly affected by oxidation conditions, rather than by water content. SEE LETTER P.355

\section{TETSUO IRIFUNE \& TOMOHIRO OHUCHI}

$\mathrm{T}$ he outermost layer of the solid Earth is divided into tectonic plates that move around on a region of the upper mantle called the asthenosphere. Seismic waves from earthquakes travel through the asthenosphere at relatively low speeds, and are highly attenuated as a result of energy dissipation, a property known as anelastic behaviour ${ }^{1}$. These seismic characteristics are usually associated with low viscosity (a 'softening') in the mantle rock peridotite. On page 355, Cline et al. ${ }^{2}$ demonstrate for the first time that this softening is influenced by oxidation conditions - a result that could have major implications for our understanding of the upper mantle.

The softening of peridotite in the asthenosphere was initially thought to be caused by small amounts of melted material ${ }^{3}$. Such material would act as a lubricant between crystals of the mineral olivine that are abundant in peridotite (Fig. 1). However, in the 1990s, it was shown that this effect is limited to particularly warm regions of the mantle, such as beneath the volcanoes that occur along mid-ocean ridges, where melted material is abundant enough to form interconnected networks ${ }^{4,5}$

Over the past two decades, the consensus has been that the presence of water leads to substantial softening of peridotite ${ }^{6}$. Experiments that measure the deformation of olivine crystals under large strains ${ }^{7-9}$ have shown that small amounts of water can enhance both the sliding of grain boundaries (the interfaces between crystals) and the deformation of individual crystals. However, the absence of appropriate equipment and techniques has meant that there have been no experiments to assess the anelastic behaviour of peridotite when it has a realistic water content (up to a few hundred parts per million ${ }^{10}$ ) and is under the small strains associated with seismic-wave propagation.

Cline and colleagues used a sophisticated method ${ }^{11}$ to subject aggregates of olivine to high temperatures and pressures, and to oscillations that mimic seismic waves known as shear waves. The authors considered aggregates that had a range of water contents and oxidation states, similar to those expected

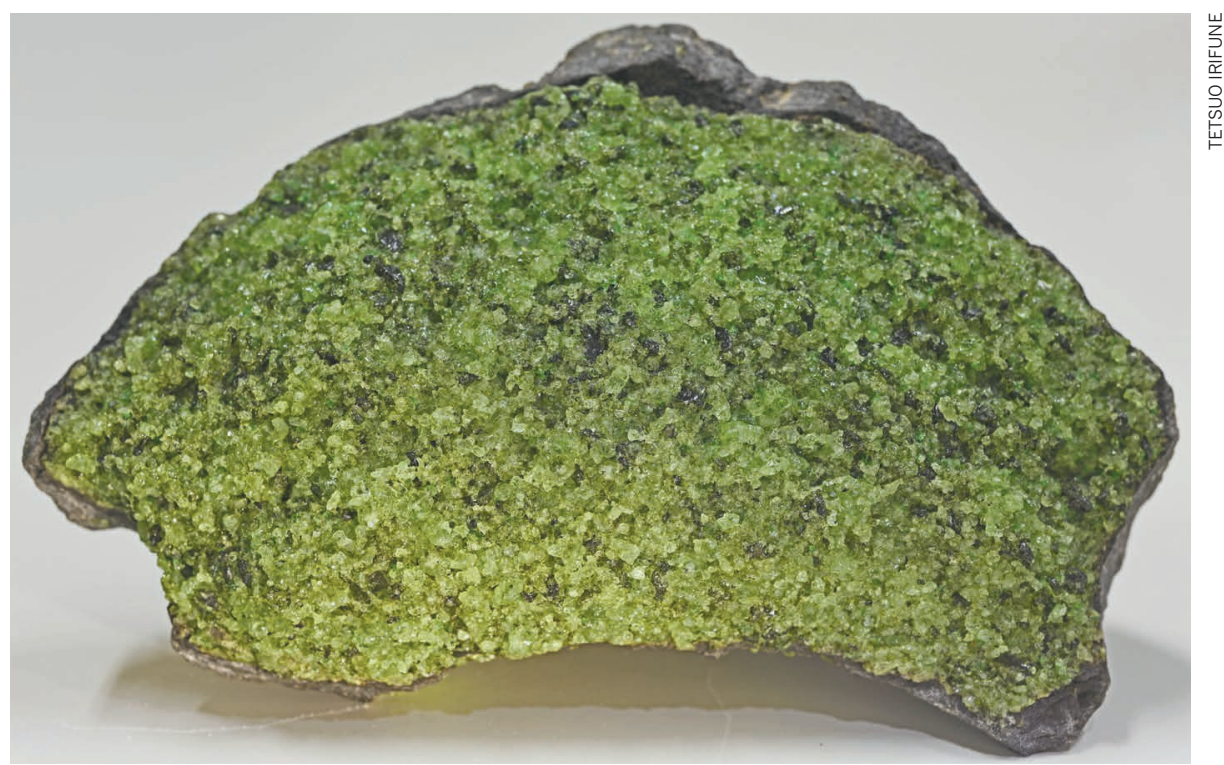

Figure 1 | Mantle peridotite from San Carlos, Arizona. Peridotite is the dominant rock in the upper part of Earth's mantle. It consists mainly of the mineral olivine (light green), with smaller amounts of other minerals such as pyroxene, spinel and garnet (darker colours). Cline et al. ${ }^{2}$ subjected aggregates of olivine to high temperatures and pressures, and to oscillations that mimic seismic waves known as shear waves. They discovered that the speed and attenuation of the waves were insensitive to the water content of olivine, but strongly dependent on oxidation conditions - findings that could reshape our view of the upper mantle.

in the asthenosphere. They discovered that the speed and attenuation of the waves were insensitive to water content, in contrast to expectations from the results of the largestrain deformation experiments ${ }^{7-9}$.

Instead, Cline et al. found that the seismic properties of their olivine aggregates were markedly dependent on oxidation state: wave speed decreased and attenuation increased with increasing oxygen fugacity (degree of oxidation). This finding could imply that the low speeds and high attenuation of seismic waves in the asthenosphere, particularly above sinking (subducting) tectonic plates, are partly caused by the highly oxidized conditions that are expected in such regions.

To explain their results, the authors suggest that ferric iron $\left(\mathrm{Fe}^{3+}\right)$ and associated metalion vacancies that exist in olivine become stabilized under oxidized conditions, yielding high concentrations of crystal defects and/or a modified grain-boundary structure. Such changes are expected to enhance the rate at which defects diffuse through the crystals, leading to the observed anelastic behaviour.

Because olivine is the most common mineral throughout the upper mantle, Cline and colleagues' findings could have implications for the oxygen fugacity and water content of not only the asthenosphere but also the entire upper mantle. For instance, if oxygen fugacity were entirely responsible for the seismic attenuation, it would have to fall by a factor of about 100 between the asthenosphere and the underlying part of the upper mantle to account for the observed decrease in attenuation ${ }^{1}$. Such a drop is consistent with petrological data from deeper regions of the upper mantle ${ }^{12,13}$.

By contrast, if attenuation were attributable to water alone, water content would need to decrease by a factor of about 100 between the asthenosphere and the underlying layer, on the basis of an earlier model ${ }^{6}$ and observations ${ }^{1}$, and peridotite at the base of the upper mantle would be almost completely dry. This prediction conflicts with observations of the mantle's electrical conductivity, which is sensitive to water content. Electrical-conductivity profiles 
are either roughly constant or increase with depth throughout the upper mantle below the asthenosphere $^{14-16}$, suggesting that water content should follow similar trends. This enigma can be solved if water is not the primary cause of seismic attenuation, as shown by Cline and colleagues.

Nevertheless, there are some issues regarding the applicability of Cline and colleagues' results to the actual mantle. For instance, the authors artificially increased the water content of some of their olivine aggregates using a technique called doping, in which a trace amount of one element is substituted for another. This process introduced artificial crystal defects whose mobility might differ from the defects intrinsic to olivine - although the authors argue that these artificial defects do not affect their conclusions. The effect of oxygen fugacity on the mobility of these different types of defect is also unknown.

Future studies on the seismic properties of olivine could avoid the need for doping by subjecting aggregates to higher pressures than those used by Cline and colleagues. For example, measurements could be made using an oscillation technique that combines a largevolume press and X-ray observations ${ }^{17}$. Future experiments should include wider ranges of oxidation conditions and olivine grain sizes than those considered by Cline et al., to confirm the dominance of oxygen fugacity over other causes of anelastic behaviour.

Although some petrological evidence suggests that oxygen fugacity in the mantle generally decreases with depth ${ }^{12,13}$, it has been difficult to evaluate how such oxidation states vary laterally. The probable link between oxygen fugacity and attenuation of seismic waves in peridotite could enable 3D mapping of oxidation states in the deep mantle, using data obtained with an imaging technique called seismic tomography. Meanwhile, the lack of correlation between water content in mantle olivine and seismic attenuation, if confirmed by independent studies at higher pressures, might require scientists to reconsider the role of water in the softening of mantle rocks, and the distribution and circulation of water throughout the deep Earth..

Tetsuo Irifune and Tomohiro Ohuchi are in the Geodynamics Research Center, Ehime University, Matsuyama 790-8577, Japan. T.I. is also at the Earth Life Science Institute, Tokyo Institute of Technology. e-mails: irifune@dpc.ehime-u.ac.jp; ohuchi@sci.ehime-u.ac.jp

1. Dalton, C. A., Ekström, G. \& Dziewoński, A. M. J. Geophys. Res. Solid Earth 113, B09303 (2008).

2. Cline, C. J. II, Faul, U. H., David, E. C., Berry, A. J. \& Jackson, I. Nature 555, 355-358 (2018).

3. Anderson, D. L. \& Spetzler, H. Phys. Earth Planet. Inter. 4, 62-64 (1970)

4. Wilcock, W. S. D., Solomon, S. C., Purdy, G. M. \& Toomey, D. R. Science 258, 1470-1474 (1992)

5. Hirth, G. \& Kohlstedt, D. L. J. Geophys. Res. Solid Earth 100, 15441-15449 (1995).

6. Karato, S. \& Jung, H. Earth Planet. Sci. Lett. $\mathbf{1 5 7}$ 193-207 (1998).

7. McDonnell, R. D., Peach, C. J. \& Spiers, C. J. J. Geophys. Res. Solid Earth 104, 17823-17845 (1999).

8. Ohuchi, T. et al. Sci. Adv. 1, e1500360 (2015).

9. Karato, S., Paterson, M. S. \& FitzGerald, J. D. J. Geophys. Res. Solid Earth 91, 8151-8176 (1986) 10. Hirschmann, M. M. Annu. Rev. Earth Planet. Sci. 34, 629-653 (2006).

11.Jackson, I. \& Paterson, M. S. Pure Appl. Geophys. 141, 445-466 (1993)

12. McCammon, C. \& Kopylova, M. G. Contrib. Mineral. Petrol. 148, 55-68 (2004).

13. Woodland, A. B. \& Koch, M. Earth Planet. Sci. Lett. 214, 295-310 (2003).

14.Sarafian, E. et al. Geochem. Geophys. Geosyst. 16 , 1115-1132 (2015).

15.Utada, H., Koyama, T., Obayashi, M. \& Fukao, Y. Earth Planet. Sci. Lett. 281, 249-257 (2009)

16. Yoshino, T., Matsuzaki, T., Yamashita, S. \& Katsura, T. Nature 443, 973-976 (2006).

17.Li, L. \& Weidner, D. J. Rev. Sci. Instrum. 78, 053902 (2007).

tissue ${ }^{5}$, and a third used radiocarbon dating to identify hippocampal-neuron turnover ${ }^{6}$. However, methodological challenges make human studies difficult to interpret, and more are required to make definitive conclusions.

Sorrells et al. set out to address this need using classic immunohistochemical techniques in which specific antibodies are bound to proteins of interest, revealing their locations in tissue. The authors used this strategy to count neural precursor cells, proliferating cells and immature neurons in samples from 59 human subjects, spanning fetal development through to old age (Fig. 1). They found streams of all three cell types migrating from an embryonic 'germinal zone' to the developing dentate gyrus at 14 weeks of gestation. By 22 weeks, migration was reduced, and immature neurons were largely restricted to the dentate gyrus. And there were many fewer immature neurons at one year of life than at earlier stages. The oldest sample containing immature neurons was taken from a 13-year-old individual. These findings are in stark contrast to the prevailing view that human hippocampal neurogenesis extends throughout adult life.

Is it possible to reconcile the findings with previous human data? Although direct comparisons are difficult, Sorrells et al. offer some explanations. For example, they find that DCX and PSA-NCAM, two proteins that reliably mark immature neurons in animals, can label mature neurons and non-neuronal glial cells in humans. Indeed, the authors show that these two markers unambiguously identify immature neurons only if both are expressed in a demands. But it was not always so. In the 1960s, biologist Joseph Altman reported 